\title{
Directional selection and developmental stability: evidence from fluctuating asymmetry of dental characters in mice
}

\author{
Larry Leamy
} Department of Biology, California State University,
Long Beach, California 90840.

Developmental stability was assessed among lines of mice subjected to 11 generations of selection for increased (up line) and decreased (down line) widths of the first maxillary molar ( $\left.\mathbf{M}^{1}\right)$, primarily to test the hypothesis that this stability would decline over time as a result of selection. Fluctuating asymmetry (FA) was used as an inverse measure of developmental stability, and was calculated for the $\mathbf{M}^{\mathbf{1}}$ and a correlated character, the second mandibular molar $\left(\mathbf{M}_{2}\right)$, in each generation. As measured by the regression of FA on generations, there was a statistically significant decline in stability only in the down selection line for the $\mathbf{M}_{2}$, and this trend did not differ significantly from that for the control line. The combined regressions obtained from pooling over all three lines were significantly different from zero in both molars, however, and this was taken to be evidence of a decline in stability due to increased homozygosity presumably generated by inbreeding during the selection experiment. The variation in $\mathrm{FA}$ among generations was greater for the selection lines than the control line for both molars, especially for the $\mathbf{M}_{2}$. The heritability of the $\mathbf{M}^{1}$ (and $\mathbf{M}_{2}$ ) was high, but there was no significant additive genetic variance for FA in either molar.

\section{INTRODUCTION}

Beginning with the early demonstrations by Lerner (1954) of the generally greater fitness of heterozygotes compared to homozygotes, there has been continued interest in the genetical basis for developmental stability or homeostasis. Lerner's original hypothesis has since been amply verified in inbred/hybrid comparisons (Leamy, 1984), but trends in developmental stability are less clear for characters in outcrossing populations or populations subjected to directional selection. Strong directional selection ought to cause a decline in stability if such selection either favours homozygous individuals or alters the population mean from its assumed optimum (Soule, 1967). The evidence on this matter has been mixed (Bradley, 1980; Leamy and Atchley, 1985), however, and more studies are needed before we can formulate general expectations for the effect of selection on developmental stability.

Developmental stability most often has been assessed by the magnitude of fluctuating asymmetry (FA) in bilateral characters (Mather, 1953; Thoday, 1958; Beardmore, 1960; Van Valen, 1962; Soule, 1979, 1982; Leamy, 1984; Leamy and Atchley, 1985). FA itself is measured in these characters by the differences between left and right sides after first correcting for the presence of any directional asymmetry. Directional asymmetry occurs when one side is significantly larger than the other, but it is the random differences remaining between sides which comprise FA. Presumably these differences are the result of a geneticallybased developmental process which tries, but nearly always fails, to produce perfect mirror images on each side (Van Valen, 1962; Bader, 1965). FA therefore is taken to be an inverse measure of developmental stability, with increased amounts of FA implying decreased developmental stability and vice versa.

The present study is concerned with assessing developmental homeostasis in a population of mice subjected to selection for the width of the first maxillary molar. FA is used as a measure of homeostasis both for this selected character as well as a correlated character, width of the second mandibular molar. The major hypothesis to be tested is that developmental stability in these characters declines during the selection process. If this is true, then we would expect an increase of FA over time in both the up and down selection lines, especially as compared to that for the control line. 


\section{MATERIALS AND METHODS}

The mice used in this study were produced in a previous two-way directional selection experiment for the width of the first maxillary molar, $\mathbf{M}^{1}$ (Larson, 1971). The genetically variable, randombred strain of house mice, Ha/ICR (Bader, 1965), was used to form the base population for the experiment. Originally, 165 individuals of this strain were obtained from Cumberland View Farms, Clinton, Tennessee. When the mice had all reached 8 weeks of age, 20 males and 20 females were chosen at random as parents of first generation offspring of the control line. The remaining mice were anaesthetised, and initial measurements of their left $\mathbf{M}^{1}$ width were taken with a binocular dissecting microscope equipped with an ocular micrometer. Those 20 mice of each sex estimated as having the widest left $\mathrm{M}^{1}$ were selected as parents for the up line. Similarly, those 20 mice of each sex having the narrowest left $\mathbf{M}^{1}$ were used as parents for the low line. Basically this same procedure was followed for 11 generations.

Single-pair matings of individuals were done systematically (Poiley, 1960) each generation in order to minimise inbreeding (Larson, 1971). In most generations, however, occasional pairs failed to produce progeny and thus 20 litters were not always available from which to draw parents of the next generation. And even when all were available, the selection process resulted in only certain families being used. As a consequence, effective population sizes in the selection lines were less than the optimal number of 40 , and the calculated rate of inbreeding (Falconer, 1981) throughout the generations averaged 0.018 in the high line, 0.017 in the low line, and 0.011 in the control line.

All litters produced each generation were standardised at birth to three males and three females where possible. Each individual was weaned by 25 days of age and sacrificed between 6 and 15 weeks of age, the precise time of sacrifice depending in part on whether the individual served as a parent for the next generation offspring. After sacrifice, all skulls were prepared by the papain digestion technique (Luther, 1949). The actual sample sizes varied from 39 to 60 for each sex in each generation of the up, control, and down lines, the total number of mice used being 3460 (1731 males, 1729 females).

Widths of both the left and the right sides of the $M^{1}$ were measured on all mice throughout the 11 generations. Additionally, left and right widths of the second mandibular molar, $\mathbf{M}_{2}$, were measured on all mice from the first 8 generations.
Measurements were taken at essentially the widest part of the teeth, these dimensions being ageconstant since the molars do not grow after they erupt into the oral cavity (Bader, 1965). All measurements were made to the nearest micron with a Gaertner traveling microscope. Their repeatibility was excellent, occasional repeat measures generally being within 6 microns of the original measurement (Larson, 1971). It should be noted, incidentally, that the analysis below is based on the results of these more precise dental measurements and not on the initial estimates of left $M^{1}$ widths used in the selection process.

Fluctuating asymmetry in the $\mathbf{M}^{1}$ and $\mathrm{M}_{2}$ widths was calculated for male and female mice in each generation in the up, control, and down lines by two separate methods. These methods yielded two measures of FA, here called mean asymmetry and variance asymmetry. To obtain mean asymmetries, right minus left differences first were calculated for each sample. The mean of these differences (in each sample) was next subtracted from each value in order to make their overall mean zero and eliminate any effects due to directional asymmetry (where one side is statistically greater than the other). Mean asymmetry values in each sex by generation by line sample ( 66 total for the $\mathbf{M}^{1}, 48$ for the $\mathbf{M}_{2}$ ) were then calculated simply by the means of the absolute differences. To correct for potential associations of the mean asymmetry values with the actual mean values of the tooth widths over all generations and lines (scaling effects, see Soule, 1967), the mean asymmetry values were appropriately adjusted via regression techniques (Sokal and Rohlf, 1981) prior to analysis.

Variance asymmetry values for each line by generation by sex combination were estimated from error variances obtained in two-way analyses of variance of the logarithmically-transformed data, the transformation being made to eliminate potential scaling effects. In these analyses of variance, sides was one factor and individuals was the second (Leamy, 1984). The sides component estimates (and effectively adjusts for) any directional asymmetry, and the error variance represents the interaction between sides and individuals and is used to estimate the variance asymmetry. This error variance presumably is generated by local environmental effects acting on the left or the right molar widths, and also is equivalent to what is known as special environmental variance (Falconer, 1981).

The variance asymmetry for each molar also may be estimated by the percentage contribution of the error variance to the total variance, this 
being an especially useful method of assessing the relative magnitude of FA (Leamy, 1984). When assessed this way, these values actually are equivalent to 1 minus the intra-class correlation, or repeatibility, between left and right sides. Repeatibilities in turn are of interest because they estimate the sum of both genetic and general environmental influences (Falconer, 1981) affecting the molars.

\section{RESULTS}

Before the results of the FA analysis are presented, it is first instructive to examine the actual means of the molars themseives throughout the generations. These are given in table 1 for the combined sexes in the up, control, and down lines. As may be seen, $M^{1}$ widths average about 1143 microns at the start of selection, increasing to 1245 microns in the up line and decreasing to 1029 microns in the down line after $1 i$ generations. The response to selection is continuous and is quite symmetric (average of 11 microns per generation for the first 8 generations in both the up and down selection lines; Larson, 1971). The correlated response to selection for the $\mathbf{M}_{2}$ also is apparent, although its magnitude over the 8 generations measured (7 microns per generation in the high line, 6 in the low line) is somewhat less than that achieved for the $\mathbf{M}^{1}$ (Larson, 1971). Further information regarding selection differentials, realised heritabilities, and other details may be found in Larson (1971), but the important point to note here is that there has been successful and substantial bidirectional change in both molars as a result of the selection process.

The variances of the tooth widths in the three lines throughout the generations also are given in table 1 in order to provide some insight into the variability of these characters. As may be seen, the variances range in magnitude from about 300 to 800 , and vary considerably from one generation to the next. Their means for the high, control, and low lines, respectively, are 539, 524, and 487 for the $\mathbf{M}^{1}$, and $53 \%$, 448, and 395 for the $\mathbf{M}_{2}$. Thus there is some indication of a scaling effect, although line and generation differences of these variances for both molars were not significantly different in analyses of variance. Further, analyses of covariance showed that regressions of variances on generations were not significantly different among the three lines in either molar, and that the combined regressions obtained from pooling over all lines were not significantly different from zero. In general, therefore, variances for both teeth are homogeneous throughout all lines and generations.

The mean asymmetry values correlated highly with the variance asymmetry values calculated in each line and generation, and it therefore was decided to use only the mean asymmetries throughout the analysis. The contributions of the variance asymmetry values to the total variance are given in table 2, however, primarily in order to provide a measure of FA variation within generations and also an assessment of the relative

Table 1. Means and variances of the $M^{1}$ and $M_{2}$ molar widths (all in microns) for mice in the up (U), control (C) and down (D) lines throughout the generations

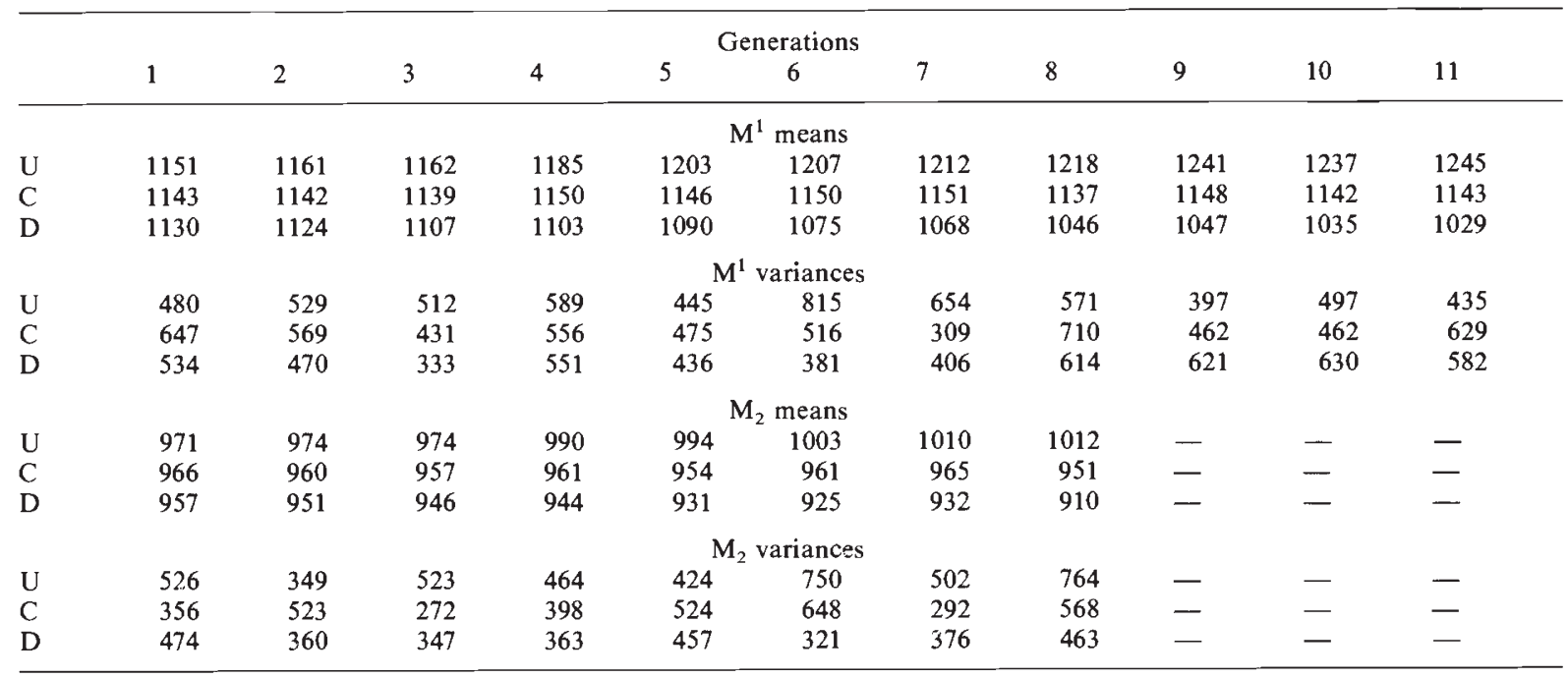


Table 2 Mean asymmetries of the $M^{1}$ and $M_{2}$ molars and their variance asymmetry percentage contribution to the total variance for mice in the up (U), control (C) and down (D) lines throughout the generations

\begin{tabular}{|c|c|c|c|c|c|c|c|c|c|c|c|}
\hline \multicolumn{12}{|c|}{ Generations } \\
\hline & 1 & 2 & 3 & 4 & 5 & 6 & 7 & 8 & 9 & 10 & 11 \\
\hline \multicolumn{12}{|c|}{$\mathbf{M}^{1}$ variance asymmetry percentages } \\
\hline $\mathrm{C}$ & $9 \cdot 9$ & $12 \cdot 7$ & $15 \cdot 1$ & $10 \cdot 9$ & $12 \cdot 5$ & $13 \cdot 1$ & $20 \cdot 6$ & $8 \cdot 7$ & $17 \cdot 3$ & $15 \cdot 8$ & $13 \cdot 1$ \\
\hline D & $9 \cdot 9$ & $16 \cdot 2$ & $17 \cdot 0$ & $11 \cdot 2$ & $14 \cdot 9$ & $19 \cdot 4$ & $12 \cdot 0$ & $12 \cdot 6$ & $11 \cdot 7$ & $10 \cdot 6$ & $12 \cdot 9$ \\
\hline \multicolumn{12}{|c|}{$\mathrm{M}^{1}$ mean asymmetries } \\
\hline $\mathrm{C}$ & $9 \cdot 3$ & $10 \cdot 2$ & $10 \cdot 4$ & $9 \cdot 5$ & $9 \cdot 1$ & $9 \cdot 9$ & $10 \cdot 4$ & $8 \cdot 9$ & $10 \cdot 8$ & $10 \cdot 7$ & $11 \cdot 0$ \\
\hline D & $8 \cdot 0$ & $11 \cdot 3$ & $9 \cdot 7$ & $9 \cdot 4$ & $9 \cdot 7$ & $11 \cdot 3$ & $9 \cdot 5$ & $10 \cdot 0$ & $10 \cdot 2$ & $9 \cdot 9$ & $10 \cdot 4$ \\
\hline \multicolumn{12}{|c|}{$\mathbf{M}_{2}$ variance asymmetry percentages } \\
\hline $\mathbf{U}$ & $8 \cdot 2$ & $12 \cdot 8$ & $10 \cdot 3$ & $13 \cdot 2$ & $14 \cdot 4$ & $12 \cdot 3$ & $12 \cdot 8$ & $8 \cdot 2$ & - & - & - \\
\hline $\mathrm{C}$ & $15 \cdot 6$ & $7 \cdot 1$ & $19 \cdot 3$ & $11 \cdot 1$ & $10 \cdot 1$ & $7 \cdot 6$ & $15 \cdot 0$ & $8 \cdot 2$ & - & - & - \\
\hline D & $7 \cdot 3$ & $12 \cdot 6$ & $13 \cdot 2$ & $13 \cdot 0$ & $12 \cdot 9$ & $12 \cdot 6$ & $12 \cdot 7$ & $10 \cdot 4$ & - & - & - \\
\hline \multicolumn{12}{|c|}{$\mathrm{M}_{2}$ mean asymmetries } \\
\hline D & $7 \cdot 1$ & $8 \cdot 0$ & $7 \cdot 9$ & $8 \cdot 2$ & $9 \cdot 7$ & $7 \cdot 7$ & 9.2 & $9 \cdot 4$ & - & - & - \\
\hline
\end{tabular}

importance of FA. Sexes were additively sexcorrected (analyses of variance did not show any significant sex interactions), so the values in the table represent the means of the two estimates in each case. As may be seen in the table, FA contributions range from about 9 to 18 percent, but average nearly the same in the up, control, and down lines for both the $\mathrm{M}^{1}(13 \cdot 3,13 \cdot 6$, and $13 \cdot 5$ per cent $)$ and $M_{2}(11 \cdot 5,11 \cdot 7,11 \cdot 8$ per cent $)$. Analyses of variance and covariance showed no significant line or generation differences or trends, and thus as was true with the variances of the molar widths themselves, the FA percentage contributions appear to be homogeneous among all lines and generations.

The mean asymmetry values for both molars in each of the lines and generations also are given in table 2 and are illustrated in fig. 1. For the $\mathbf{M}^{\mathbf{1}}$, these values range from 8 to nearly 12 microns, their overall average being nearly 10 . All lines appear to show a general increase in FA through time, especially during the last 3 generations. The range for the $M_{2} F A$ values is somewhat more restricted (approximately 7 to 9 microns) and their average is slightly over 8 microns. Their magnitude is consistent in the control line (except for the drop to 7.1 microns in generation 2), but generally tends to increase in the up and especially the down selection line.

These trends are quantified in table 3 where the means and variances of the mean FA values over generations, as well as their regressions on generations, are given for each line. As for trends over time, the positive sign of all six individual regressions indicates that FA has generally increased through the generations. The values are highest in magnitude for the $\mathbf{M}_{2}$ in both selection lines, especially that for the down line $(0 \cdot 27)$ which in fact is the only one significantly different from zero $(P<0.05)$. However, analyses of covariance indicated that comparisons of these regressions among all three lines, between up versus down lines, and between both selected lines versus the controls, all were non-significant for both molars. Common regressions were therefore obtained by pooling over all lines, and these were significantly different from zero $(P<0.05)$ for both the $\mathbf{M}^{1}$ $(0 \cdot 10)$ and $M_{2}(0 \cdot 14)$. Thus developmental stability in both molars definitely appears to have decreased with time, but across all lines, control and selection alike.

Although trends in the FA values over time are of principal importance in this study, patterns in the means and variances of these values among the lines also are of some interest. As may be seen in table 3, the averages of the mean FA values for both molars are quite similar in all three lines, so it is apparent that the overall mean level of fluctuating asymmetry has not been affected by selection. Variances of the mean FA values among generations are greater in the selection lines than in the control line for both molars, however, both the up versus control and down versus control variance comparisons being significant $(P<0.05)$ for the $\mathrm{M}_{2}$, but not $(P=0 \cdot 10)$ for the $\mathrm{M}^{1}$. FA variability therefore apparently has been affected by selection, at least for the $\mathbf{M}_{2}$. 


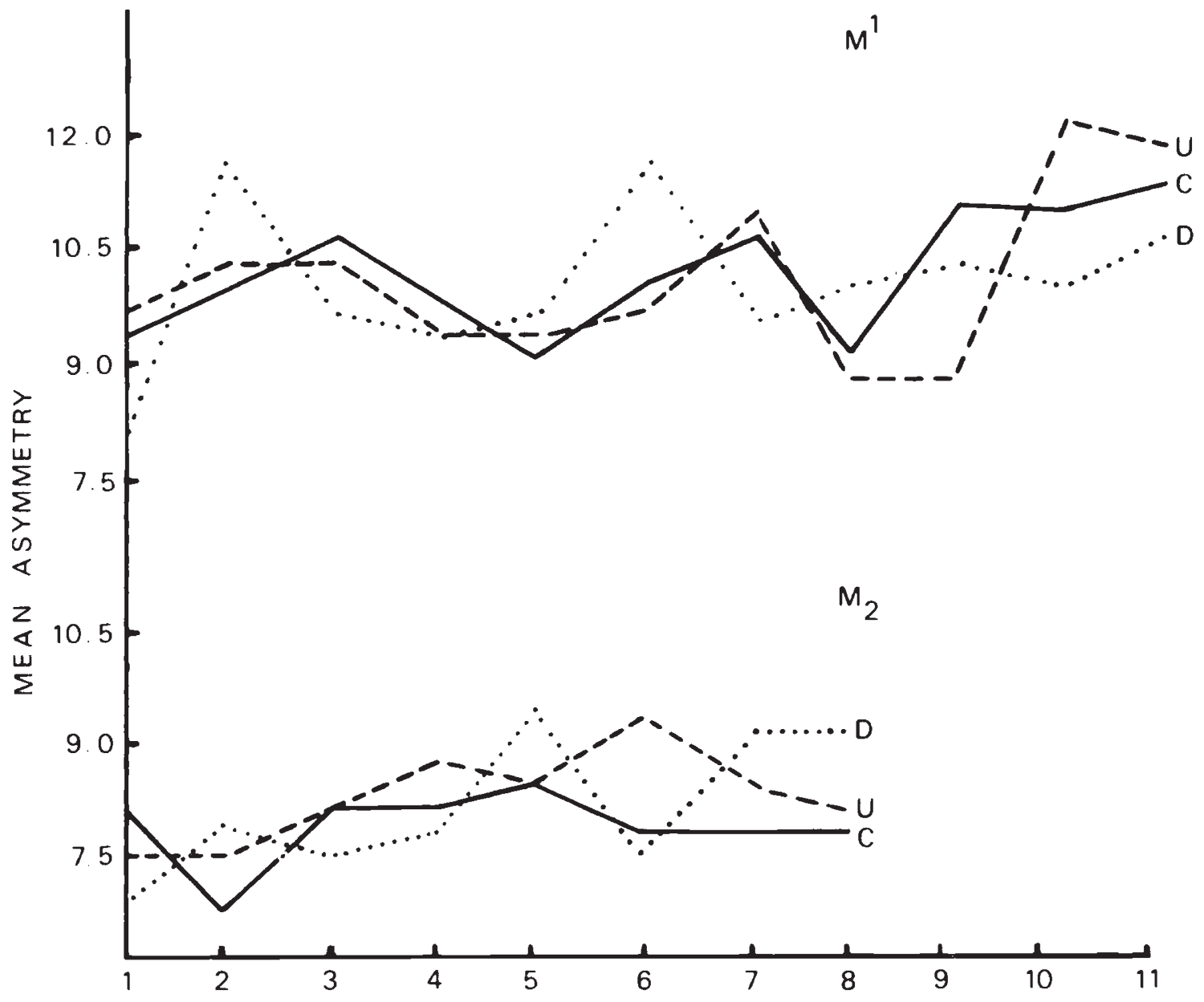

\section{GENERATIONS}

Figure 1 Mean fluctuating asymmetry for the $\mathrm{M}^{1}$ and $\mathrm{M}_{2}$ in the up (U), control (C), and down (D) line over the generations.

Table 3 Means $(\overline{\mathrm{X}})$ and variances $\left(s^{2}\right)$ of the $\mathrm{M}^{1}$ and $\mathrm{M}_{2}$ asymmetries and regressions $(b)$ of these asymmetries on generations (standard errors in parentheses) in the up, control, and down lines, and for all pooled lines

\begin{tabular}{|c|c|c|c|c|}
\hline & Up & Control & Down & Pooled lines \\
\hline \multicolumn{5}{|c|}{$\mathrm{M}^{1}$ asymmetry } \\
\hline$\overline{\mathbf{X}}$ & $9 \cdot 94$ & 9.99 & 9.95 & $9 \cdot 96$ \\
\hline$s^{2}$ & 1.48 & 0.82 & $1 \cdot 35$ & $1 \cdot 22$ \\
\hline$b$ & $0.12(0.080)$ & $0.12(0.062)$ & $0.08(0.078)$ & $0 \cdot 10^{*}(0.040)$ \\
\hline \multicolumn{5}{|c|}{$\mathrm{M}_{2}$ asymmetry } \\
\hline$\overline{\mathrm{X}}$ & $8 \cdot 54$ & $8 \cdot 19$ & $8 \cdot 42$ & $8 \cdot 38$ \\
\hline$s^{2}$ & $1 \cdot 31$ & 0.52 & $1 \cdot 37$ & 1.07 \\
\hline$b$ & $0.14(0.123)$ & $0.01(0.081)$ & $0.27^{*}(0.110)$ & $0.14^{*}(0.062)$ \\
\hline
\end{tabular}

$*=P<0.05$. 
The analysis of variance of the mean FAs in both molars is given in table 4. As was inferred earlier from the overall means, FA does not significantly differ among the three lines (or for the selection versus control line comparisons) for either molar. Further, since the lines $\times$ generations interactions are not significant, FA trends over generations apparently are parallel among the lines. Again, this is consistent with the previous results of analysis of covariance of the regressions. FA in the $\mathbf{M}^{1}$ (but not $\mathbf{M}_{2}$ ) among generations is statistically significant, and this is probably because of the wide variation among the means from one generation to the next (see table 2 and fig. 1) more so than the overall positive upward trend.

Table 4 The analysis of variance for the $M^{1}$ and $M_{2}$ mean asymmetries

\begin{tabular}{lcll}
\hline & & \multicolumn{2}{c}{ Mean squares } \\
Source & df & $\begin{array}{l}\mathbf{M}^{\mathbf{1}} \\
\text { asymmetry }\end{array}$ & $\begin{array}{l}\mathbf{M}_{2} \\
\text { asymmetry }\end{array}$ \\
\hline Lines & 2 & 0.018 & 0.513 \\
$\quad$ Up versus Control & 1 & 0.029 & 0.995 \\
Down versus Control 1 & 0.025 & 0.426 \\
Generations & 10,7 & $2 \cdot 796^{* *}$ & $1 \cdot 225$ \\
Lines $\times$ Generations & 20,14 & $1 \cdot 136$ & 0.865 \\
Error & 33,24 & 0.781 & $1 \cdot 131$ \\
\hline
\end{tabular}

In order to discover whether the FA trends were associated either between molars or lines, the appropriate correlations were calculated and are displayed in table 5. Correlations between FAs in the $M^{1}$ and $M_{2}$ over the first 8 generations are not statistically significant in any of the three lines. Correlations of the $\mathrm{M}^{1}$ asymmetries (11 generations) and $\mathbf{M}_{2}$ asymmetries (8 generations) for each pair of lines show significance only for the up-control combination for $\mathbf{M}^{1}$. Except for this

Table 5 Correlations of $M^{1}$ and $M_{2}$ asymmetries in the up, control, and down lines, and correlations of the asymmetry in each tooth in the up-control, down-control, and up-down line combinations

\begin{tabular}{|c|c|c|c|}
\hline & Up & Control & Down \\
\hline \multirow{2}{*}{$\begin{array}{l}\mathbf{M}^{1}-\mathbf{M}_{2} \\
\text { asymmetries }\end{array}$} & 0.15 & -0.12 & $0 \cdot 21$ \\
\hline & $\begin{array}{l}\text { Up- } \\
\text { control }\end{array}$ & $\begin{array}{l}\text { Down- } \\
\text { control }\end{array}$ & Up-down \\
\hline $\mathbf{M}^{1}$ asymmetry & $0.43^{*}$ & 0.07 & $0 \cdot 10$ \\
\hline $\mathrm{M}_{2}$ asymmetry & 0.03 & 0.00 & $-0 \cdot 40$ \\
\hline
\end{tabular}

instance, however, in general there is little association of the FA values either between molars or lines.

\section{DISCUSSION}

The basic purpose of this experiment was to discover whether developmental stability in the widths of the two molars in this population of mice has decreased as a result of selection. As inversely measured by fluctuating asymmetry, there was such a decline in stability in both molars, but it occurred for the control line as well as the selected lines in the molar subjected to direct selection, the $\mathbf{M}^{1}$. Trends for the $\mathrm{M}_{2}$ were much more suggestive in that both selected lines showed a greater increase in FA over time (pooled regression $=0.21 ; P<$ $0.05)$ compared to that for the control line (regression $=0.01$ ), but the comparison itself (of selection versus control line regressions) was not statistically significant. We are thus left with the conclusion that developmental stability in both molars has declined through the several generations, but this decline is common to both the selection and control lines.

Although there clearly was an increase through time in the magnitude of FA in the selection and control lines, it must be borne in mind that this pattern is based on sampling from a relatively few generations. Even though a rather large total number of mice of both sexes was used, sampling variation can certainly affect trends based on the means of the FA values at each generation. Inspection of the control line mean FA values for $\mathbf{M}^{1}$ (table 2), for example, shows that their upward trend is primarily due to high values in the last three generations; in fact, the regression of FAs on the first 8 generations only is negative in sign $(-0.044)$. These three values also undoubtedly have contributed to the somewhat higher variability of the control line FAs in this molar (compared to the $\mathrm{M}_{2}$ ), to the significant overall variation among generations detected in the analysis of variance (table 4), and to the positive correlation of FAs between the control and up lines (table 5).

It is reasonable to believe that the regression of FA on generations ought to be rather low in magnitude in the control line, because developmental stability should remain roughly constant or even increase (and thus FA decrease) with time in a population subjected only to natural selection (Soule, 1979). There was some unconscious selection for larger $\mathrm{M}^{1}$ widths themselves in the control line (see Larson, 1971), but it was very small in 
magnitude compared to that actually practiced in the up line and should have had little or no effect on FA. Chaeta numbers and their FA did significantly decline in the control population of Drosophila used by Bradley (1980), especially over the last 20 compared to the first 20 generations.

It was originally hypothesized that FA in both molars in the up and down selection lines should increase (and developmental stability decrease) if in fact the selection process has produced increased homozygosity which in turn is negatively associated with development stability (Mitton, 1978; Soule, 1979; Soule and Cuzin-Roudy, 1982; Leamy, 1984; Livshits and Kobyliansky, 1985). Fixation of alleles leading to increased homozygosity is one expected theoretical result of directional selection (Falconer, 1981), although it is difficult to say how important this has been in the mouse selection lines. The selection limit has not yet been reached here, and also the heritabilities of both molars remained consistently high through the generations (Larson, 1971), so it possible that the differences in homozygosity between the selection and control lines are not as great as expected.

On the other hand, there seems little doubt that inbreeding has been a significant factor in producing homozygosity, probably for all three lines. Robertson (1961) has shown that selection acts to reduce the effective size of a population and thus increase the rate of inbreeding, and that this effect is expected to be greater when the selection criterion has a high heritability. The heritability of the width of the $\mathrm{M}^{1}$ was rather high (average of $0.6)$ throughout the generations (Larson, 1971), and in fact this was reflected in the substantial rates of inbreeding previously given. To see whether there was a significant association of inbreeding and FA, regressions of the FA values on the inbreeding coefficients ( F values $\times 100$ ) were calculated for each line. For the up, control, and down lines, these values were $0.07,0.07$, and 0.09 for the $M^{1}$ and $0.09,0.01$, and 0.26 for the $M_{2}$. The three regressions for each molar were not significantly different, so common regressions were calculated for the $\mathbf{M}^{1}(0.07)$ and $\mathbf{M}_{2}(0.09)$, both of which were significantly different from zero $(P<0.05)$. Basically these values parallel those for the regressions of FA on generations, and suggest that inbreeding did indeed have a significant effect on the level of FA throughout the generations.

It should be mentioned that a number of studies (for example, Soule, 1967) have shown that the effects of homozygosity on bilateral characters are relatively general; that is, their FA seems to increase with homozygosity at most loci. Many populations in fact seem to have a characteristic level of FA among all of their bilateral characters, a level which may differ considerably from that of other populations (see discussion of the "population asymmetry parameter" in Soule, 1967; Leamy, 1984). In the selection experiment here, the two molars share many genes in common since the genetic correlation between them was consistently high (average of about $+0 \cdot 7$ ) throughout the generations and lines (Larson, 1971). However, a similar decline in FA through the generations might have been expected even for other bilateral characters not pleiotropically related to $\mathbf{M}^{1}$.

Perhaps as important as the general increase of FA was its greater variability throughout the generations in the selection lines compared to the controls, especially for the $\mathbf{M}_{2}$. What this suggests is that directional selection somehow has influenced FA (and therefore developmental stability) in these molars, causing its magnitude to vary more from one generation to the next. This is not merely a scaling effect, incidentally, because regression adjustments were made in the calculation of the FA values; further, the pattern of change in these values throughout the generations is mainly one of randomness (fig. 1). In an experiment with Drosophila, Bradley (1980) found greater amonggeneration variation of FA of chaeta number in lines subjected to natural selection (where chaeta number declined) than in lines subjected to stabilising selection (where chaeta number remained constant). It therefore is possible that increased variability of FA among generations may well be one of the consequences of directional selection, but more experiments will be needed to discover whether this is generally true.

It was interesting that in the face of the increased between-generation variation of FA in the selection lines as well as the general increase of mean and variance FA in all lines, the relative contribution of the (variance) asymmetry to the total variance was homogeneous throughout the lines and generations. In part this is no doubt a consequence of the considerable variation among the variances of the molars themselves (see table 1), the FA percentage contributions to these total variances also reflecting this variability (table 2). Repeatibilities obtained for both molars by subtracting these values from 1 also naturally exhibited the same amount of variation among the lines and generations.

General environmental variances (Falconer, 1981) calculated by subtracting additive genetic variance estimates from the repeatibilities in the up, control, and down lines, averaged $19 \cdot 8,27 \cdot 0$, 
and $34 \cdot 0$ per cent for the $M^{1}$, and $29 \cdot 4,44 \cdot 3$, and 39.4 per cent for the $M_{2}$. In analyses of variance for both molars, these values did not significantly differ between lines or generations. Regressions of the general environmental variances on generations were $-2 \cdot 1,-4 \cdot 4$, and $1 \cdot 7$ for the $M^{1}$ and $-4 \cdot 1,-2 \cdot 8$, and $-7 \cdot 5$ for the $M_{2}$, analyses of covariance showing that only the regression obtained by pooling the $M_{2}$ values for all three lines was significantly different from zero $(P<$ $0.05)$. There obviously is some tendency for the general environmental variances to decline with time, however, in direct contrast to the special environmental variances (raw error variances themselves) which generally increased throughout the generations.

Although bilateral characters such as teeth often are significantly heritable, usually the heritability of the differences between their sides (FA) is zero or very low (Reeve, 1960; Leamy, 1984; Maynard Smith et al., 1985). Regressions of offspring on midparent (Falconer, 1981) were used to estimate heritabilities of FA here, and for the up, control, and down lines, averaged $0.01,-0.02$, and 0.11 for the $M^{1}$, and $-0.04,0.05$, and -0.01 for the $\mathbf{M}_{2}$. In spite of the rather small standard errors associated with these estimates (about 0.05 to 0.06 ), none reached statistical significance. Thus like most other characters so analysed, the molar widths show no significant additive genetic variation for FA. Maynard Smith et al. (1985) have suggested that the lack of genetic variation in a trait may be taken as evidence that it is under a developmental constraint. Whatever the actual case, it is apparent that much remains to be learned about the genetic and environmental interplay responsible for the creation and change in FA and developmental stability during selection.

Acknowledgments I should like to thank Joan Larson for providing the data on which this paper is based, Michael Soule for helpful comments on an early draft of the paper, Susan Guizar for drawing the figure, and James Cheverud and an anonymous reviewer for useful revision suggestions. This work was supported in part by a faculty grant from the University Research Committee, California State University, Long Beach.

\section{REFERENCES}

BADER, R. S. 1965. Fluctuating asymmetry in the dentition of the house mouse. Growth, 29, 291-300.

BEARDMORE, J. A. 1960. Developmental stability in constant and fluctuating temperatures. Heredity, 14, 411-422.

BRADLEY, B. P. 1980. Developmental stability of Drosophila melanogaster under artificial and natural selection in constant and fluctuating environments. Genetics, 98, 10331042.

FALCONER, D. S. 1981. Introduction to Quantitative Genetics. Longman, New York.

LARSON, M. J. 1971. Selection for an odontometric trait in a genetically variable strain of the house mouse. Ph.D. Thesis, University of Illinois, Urbana.

LEAMY, L. 1984. Morphometric studies in inbred and hybrid house mice. V. Directional and fluctuating asymmetry. American Naturalist, 123, 579-593.

LEAMY, L. AND ATCHLEY, w. 1985. Directional selection and developmental stability: Evidence from fluctuating asymmetry of morphometric characters in rats. Growth, 49, 8-18.

LERNER, I. M. 1954. Genetic Homeostasis. Oliver and Boyd, London.

LIVSHITS, G. AND KOBYLIANSKY, E. 1985. Lerner's concept of developmental homeostasis and the problem of heterozygosity level in natural populations. Heredity, 55, 341-353.

LUTHER, P. G. 1949. Enzymatic maceration of skeletons. Proc. Linn. Soc, 161, 146-147.

MATHER, K. 1953. Genetical control of stability in development. Heredity, 7, 297-336.

MAYNARD SMITH, J. BURIAN, R. KAUFFMAN, S. ALBERCH, P., CAMPBELL, J. GOODWIN, B. LANDE, R., RAUP, D. AND WOLPERT, L. 1985. Developmental constraints and evolution. The Quarterly Review of Biology, 60, 265-287.

MITTON, J. B. 1978. Relationship between heterozygosity for enzyme loci and variation of morphological characters in natural populations. Nature, 273, 661-662.

POILEY, S. J. 1960. A systematic method of breeder rotation for non-inbred laboratory animal colonies. Proc. Animal Care Panel, 10, 159-166.

REEVE, E. C. R. 1960. Some genetic tests on asymmetry of sternopleural chaeta number in Drosophila. Genetical Research, 1, 151-172.

ROBERTSON, A. 1961. Inbreeding in artificial selection programmes. Genetical Research, 2, 189-194.

SOKAL, R. R. AND ROHLF, F. J. 1981. Biometry, 2 nd edition. Freeman, San Francisco.

SOUlE, M. 1967. Phenetics of natural populations. II. Asymmetry and evolution in a lizara. American Naturalist, 101, 141-160.

SOULE, M. 1979. Heterozygosity and developmental stability: another look. Evolution, 33, 396-401.

SOULE, M. 1982. Allomeric variation. 1. The theory and some consequences. American Naturalist, 120, 751-764.

SOULE, M., AND CUZIN-ROUDY, J. 1982. Allomeric variation. 2. Developmental instability of extreme phenotypes. American Naturalist, 120, 765-786.

THODAY, J. M. 1958. Homeostasis in a selection experiment. Heredity, 12, 401-416.

VAN VALEN, L. 1962. A study of fluctuating asymmetry. Evolution, 16, 125-142. 